\title{
Vesicourethral anastomosis including rhabdosphincter in retropubic radical prostatectomy: Technique and results
}

\author{
Ramazan Topaktaş ${ }^{1}$, Ahmet Ürkmez ${ }^{1}$, Musab Ali Kutluhan ${ }^{1}$, İsmail Başıüyük ${ }^{2}$, Şinasi Yavuz Önol ${ }^{3}$ \\ ${ }^{1}$ Haydarpaşa Numune Training and Research Hospital, Department of Urology, Istanbul, Turkey; \\ ${ }^{2}$ Beylikdüzü Kolan Hospital, Department of Urology, Istanbul, Turkey; \\ ${ }^{3}$ Onol Urology Center, Department of Urology, Istanbul, Turkey.
}

\begin{abstract}
Summary Objective: Retropubic radical prostatectomy $(R R P)$ is still widely used in clinical practice in localized prostate cancer because of its high oncological success. The aim of this study was to define the continence status in patients where rhabdosphincter was included in the vesicourethral anastomosis.

Materials and methods: Between November 2004 and

September 2010, 90 cases who underwent RRP by the same surgeon in our clinic were taken into the study. In all cases vesicourethral anastomosis was performed include the rhabdosphincter. The anastomosis was performed with mean 2.9 (0-7) interrupted no 2-0 vicryl sutures, depending on the angulation of symphysis pubis and pelvic cavity. Pad test was performed to all patients at 1, 3, 6 and 12 months postoperatively. We defined patients as 'continent' when they no need pad, as 'mild incontinence' when they use only one pad daily, as 'moderate incontinence' when they use two or three pads daily and as 'severe incontinence' when they use more than three pads daily. Results: Preoperative total PSA value was $12.2 \mathrm{ng} / \mathrm{ml}$ (range: 2.7-84 $\mathrm{ng} / \mathrm{ml}$ ). Preoperative prostate biopsy results were found that Gleason scores were 5, 6, 7 and 8 in 7, 53, 21, 9 patients, respectively. Mean operation and urethral catheter removal time was 103 minutes (60-200) and 14,6 days (9-28), respectively. Mean hospital stay was 4.6 days (2-20). According to results of postoperative pad tests, 38 (42.2\%), 48 (53.3\%), 55 $(61.1 \%)$ and $75(83.3 \%)$ patients were defined as continent in first, third, sixth and twelfth months, respectively.

Conclusions: We think that, our novel technique of vesicourethral anastomosis in standard RRP provides more optimal urethral position during fixation of pelvic floor and urethra, protect caudal retraction, preserve functional urethral length. Also strong full thickness stitch on urethra provides better urinary continence by hanging urethra in our patients. Although our early continence rate is better, our long term continence rate is similar to literature.
\end{abstract}

KEY WORDS: Retropubic radical prostatectomy; Vesicourethral anastomosis; Incontinence; Rhabdosphincter.

Submitted 3 June 2018; Accepted 19 July 2018

\section{INTRODUCTION}

Prostate cancer is common in man and is the second most common cause of death after lung cancer. Incidence of prostate cancer incline after 50 years old and change in different countries according to diet, ethnicity, life style and screening protocols (1).
Prostate cancer is organ defined in almost $40 \%$ of cases and gold standard treatment is radical prostatectomy (RP) if patient has ten-year life expectancy (2). After 1970s this procedure has been standard treatment especially after Walsh's contributions (3). Although this surgical procedure has important efficiencies it has also morbidities that should be considered. To get rid of morbidities and increase efficiency some modified techniques have been tried over years. Although in recent decades, laparoscopic and robot-assisted laparoscopic RP have been utilized as alternatives to traditional open surgery, RRP is still widely used in clinical practice (4).

In all surgical techniques main aim is to get oncologic control and at the same time to maintain erectile function and continence which otherwise affected can decrease patient quality of life.

Perioperative and post-operative early complications of radical prostatectomy are bleeding, rectal injury, deep venous thrombosis, pulmonary emboli and lymphocele. Late complications are urinary incontinence, erectile dysfunction and anastomotic stricture. Most important complication that affect quality of life is urinary incontinence. According to studies incidence is between 5\%$30 \%(5,6)$. Although older studies indicate higher incidence of urinary incontinence in recent years with better knowledge about pelvic anatomy this incidence has decreased.

Vesicoureteral anastomosis is one of the most important step in radical prostatectomy and if it is not done properly it leads urinary leak, urine accumulation in surgical field and prolonged drainage (7). On the other hand, it leads periurethral fibrosis, bladder neck stricture and urinary incontinence (8).

Recently improvements in techniques of vesicoureteral anastomosis provides decrease in incidence of stricture and incontinence. Urinary continence recovery is a fundamental goal for patients RRP and several surgical techniques, mainly based on musculofascial posterior and/or anterior ligaments reconstructions, have been proposed with the aim of improving urinary continence recovery.

Aim of our study is to evaluate post-operative urinary incontinence in patients who underwent RRP and had vesicoureteral anastomosis that includes rhabdosphincter. 
Figure 1.

Suture that involves the rhabdospinchter.

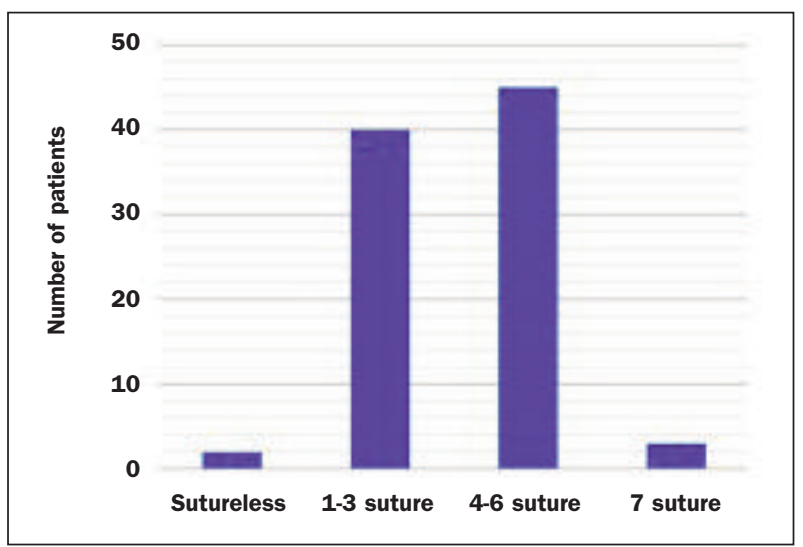

\section{Patients And methods}

Ninety patient who underwent RRP because of clinically localized prostate cancer between November 2004 and September 2010 in Bezmialem Vakıf University Medicine faculty hospital urology clinic was included in our study. Local ethics committee approval was taken for study and written consent for each patient was also taken. Preoperative and postoperative information of all patient was recorded prospectively. Exclusion criteria were: previous urethral or prostatic endoscopic procedures, preoperative urinary incontinence and concomitant neurological diseases (e.g. Parkinson disease). Bone scan was performed for intermediate and high-risk prostate cancer group and also patients who were symptomatic. In some patients MRI was used for confirmation of bone scan lesion. One experienced surgeon performed all surgeries and bladder neck preserved as much as possible. When indicated lymph node dissection was performed and in proper patients nerve sparing technique was used 2-0 vicryl suture was used in vesicourethral anastomosis. Sutures placed on urethra including rhabdospinchter from out to in and in to out for bladder (Figure 1). $22 \mathrm{~F}$ Foley catheter was placed. A watertight test was performed at the end of the procedure.

Age, psa levels, prostate volumes and prostate biopsy results of all patients were recorded preoperatively. Operation time, urethral catheterization time, retrivel of drenage time, hospital stay, suture number for vesicoureteral anastomosis and perioperative bleeding was recorded for all patient. Also, post-operative follow up period, pathological parameters and complications were recorded. For all patients postoperative third-generation cephalosporin, low-molecular-weight heparin, and elasto-compressive stockings were used for prophylaxis of infections and thromboembolic events, respectively. In postoperative period patients were followed up regularly for urinary incontinence. After retrieval of urethral catheter pad test were used for evaluation of urinary incontinence in 1, 3 and 12 months. Patients who didn't use pad in a day described as continent, patients who used one pad a day described as mild incontinent, patients who used 2-3 pad a day described as moderate incontinent and patients who used 4 pad and more described as severe incontinent.
Table 1.

Preoperative clinical features, operative and early postoperative results of patient.

\begin{tabular}{|lc}
\hline & $\begin{array}{c}\text { Mean } \pm \text { Standard deviation } \\
\text { (distribution) or number }\end{array}$ \\
\hline Total number of patients & 90 \\
\hline Age (year) & $64.3 \pm 6.01(51-78)$ \\
\hline Prostate volume (ml) & $49.2 \pm 24.18(20-160)$ \\
\hline Preoperative serum PSA level (ng/ml) & $12.2 \pm 9.54(2.7-84)$ \\
\hline Preoperative Gleason score & $6.3 \pm 0.42(5-8)$ \\
\hline Operation time (minute) & $103 \pm 52(60-200)$ \\
\hline Intraoperative bleeding (ml) & $780 \pm 723.25(150-3500)$ \\
\hline Drainage time (day) & $4.3 \pm 2.82(2-19)$ \\
\hline Urethral catheterization time (day) & $14.6 \pm 4.39(9-28)$ \\
\hline Hospital stay (day) & $4.6 \pm 7.89(2-29)$ \\
\hline Mean follow up (month) & $15.3 \pm 2.25(14-25)$ \\
\hline
\end{tabular}

\section{RESULTS}

Patient number, age, prostate volume, preop PSA values, prostate biopsy results, surgical parameters, hospitality and follow up period were summarized in Table 1. $48.8 \%$ (44) of patient had history of cigarette smoking. Mean operative bleeding was $780 \mathrm{ml}(150-3500 \mathrm{ml})$ and $32,2 \%$ (29) of patient had blood transfusion.

In one patient myocardial infarction occurred in postoperative second day and transferred to coronary intensive care unit. In this patient urethral catheter was taken off at postoperative $28^{\text {th }}$ day. Average suture number for vesicourethral anastomosis was 2.9 (0-7) and in two patient there was no suture (Figure 2). In three patients (3.3\%) acute urinary retention occurred after urinary catheter retrieval and these patients were catheterized with $12 \mathrm{~F}$ catheter for three more day. In four patients (4.4\%) rectal damage occurred in operation and primer reconstruction with two layers without colostomy was performed. In one patient omental flap with pedicul was used for strengthening rectal repair. In one patient during bladder neck dissection ureteral damage occurred and repaired intraoperatively with ureteroneocystostomy.

\section{Figure 2.}

Suture numbers for vesicourethral anastomosis.

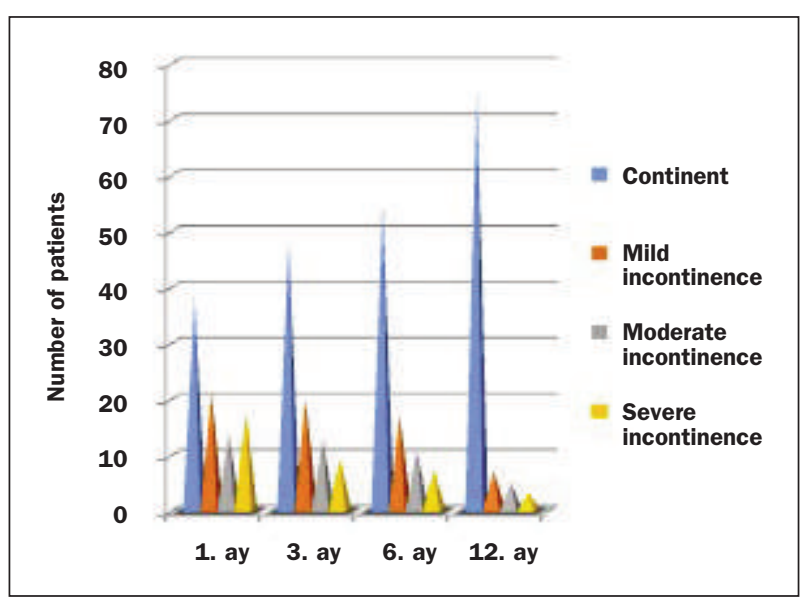




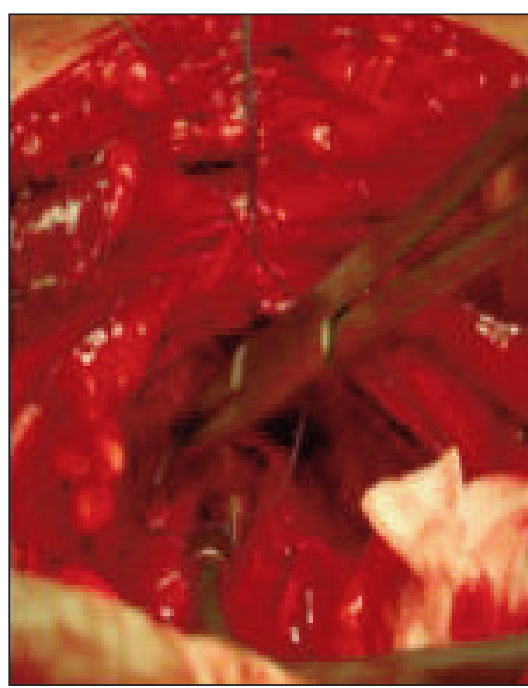

Figure 3.

Pad test classification according to months after catheter removal.

After catheter retrivel bladder neck stenosis developed in 10 patients $(11,1 \%)$ after a mean of 4.3 months (range 2 7 months) and treated with endoscopic incision. According to pathologic reports $13(14.4 \%)$ patient had pT2a, 15 (16.6\%) had pT2b, 28 (31.1\%) had pT2c, 27 (30\%) had pT3a and 7 (7.7\%) patient had pT3b. On the other hand, $6(6.6 \%)$ patient had positive surgical margin and 84 (93.3\%) patient had negative surgical margin. In 6 patients with surgical margin positivity, 4 positivity was in prostatic apex while 2 positivity was in other regions of the prostate

At first year of follow up 75 (83.3\%) patient was continent, 7 (7.7\%) patient had mild urinary incontinence, 5 (5.5\%) patient had moderate urinary incontinence and 3 (3.3\%) patient had severe urinary incontinence.

The continence status of the patients according to the postoperative month was summarized in Figure 3.

Seven patients with moderate incontinence and three patients with severe incontinence were found to have endoscopic procedures due to bladder neck stenosis after RRP at the first year of follow-up

\section{Discussion}

The most important factor that affect patient's quality of life after RRP is urinary continence status and early recovery of continence increases patient satisfaction (9). Although there was high rate of urinary incontinence in first years of radical prostatectomy prevalence has decreased over years with better understanding of pelvic anatomy, increase in experience, technology and surgical techniques. Although there are many studies that try to explain which factors like nerve sparing surgery, tabularization of bladder neck, anastomosis suture number and laparoscopic continuous suturing effect post prostatectomy incontinence, we still can't estimate which patient has urinary incontinence after radical prostatectomy (1012). One of the most important step in radical prostatectomy is vesicourethral anastomosis. General principles for good anastomosis are watertight, non-stretch, anastomoses that provide the best urethral length and mucosa to mucosa anastomosis
Several surgical techniques have been proposed in the recent years, with the aim of reducing the urinary continence recovery time and/or improving long-term urinary continence rates. Some authors indicated that nerve sparing surgery had positive affect on recovery of urinary continence (12). On the other hand, few studies indicated that nerve sparing surgery had no effect on urinary continence (13). In summary nerve sparing surgery has positive contribution on urinary continence. In our study we did not evaluate the nerve-sparing technique variable in our study this deficiency can cause misconceptions.

Rhabdospinchter is major structure that influence continence anatomically. It extends like $\Omega$-shaped from membranous urethra to prostatic apex anterolaterally. In normal conditions, the urethral sphincter is supported anteriorly by puboprostatic and pubourethral suspensory components, laterally by the medial portion of the levatori ani muscle forming a hammock around the urethra, and by the ischioprostatic ligaments. In our study we placed anastomotic sutures deeply unlike the traditional RRP described by Walsh especially in anterior urethra where intense rabdosphincter that surrounds urethra exist (3). We suppose that anastomotic suture that includes rhabdosphincter provides almost original urethral length, prevent caudal retraction of urethra and provide better anatomic positioning of urethra and bladder on pelvic floor. In patients with our technique early functional results are better than literature but long-term outcomes are same. Proper and well done apical dissection leads to a better appearance of rhabdosphincter by protecting it. In 2005 Montorsi et al. demonstrated that well done apical dissection in nerve sparing surgery protects rhabdosphincter and after catheter retrieval 44\% of patient was continent (14). In our study apical surgical margin positivity was seen only in four (4.4\%) patient. Prevalance of apical surgical margin positivity in literature is between $6.5 \%-38 \%$ (15). We think that well done apical dissection for protecting rhabdospincter provides better continence status.

Rocco et al. stated that restoration of rhabdosphincter posteriorly or in other word placing Rocco sutures provides retraction of rhabdospinchter caudally and prevents separation at posterior median raphe (16). In this study 250 patient who underwent posterior rhabdospinchter repair were compared to 50 patient who didn't. At the third month of follow up incontinence rate was $85.2 \%$ in first group who underwent rhabdospincter repair while it was $46 \%$ in control group (16). Long term incontinence rate was same in both groups (94\% vs 90\%) and rhabdospinchter repair didn't cause additional complication.

There are conflicts in studies which evaluate the relationship between incontinence and intraoperative hemorrhage. In some studies, there was no relationship between intraoperative hemorrhage and post prostatectomy incontinence. On the other hand, some studies reported that there was significant relationship between intraoperative hemorrhage and post prostatectomy incontinence $(13,17)$. Intraoperative hemorrhage alone is not a predictive factor for post prostatectomy incontinence without evaluation of other surgical factors. In our 
study at first year of follow up only 2 patients out of 15 patient who had mild, moderate and severe incontinence had more than $1000 \mathrm{cc}$ intraoperative bleeding.

Some studies stated that urethral length should be kept as long as possible for the recovery of continence after RRP and too many sutures for anastomosis shortens urethral length (18). Additionally, some studies indicated that less suture number for vesicourethral anastomosis had positive effects on urinary continence and bladder neck stenosis (19). In our study mean suture number was $2.9(0-7)$. We think that too many sutures for anastomosis has negative effects on urethral length.

Another topic related to continence is preservation of bladder neck in radical prostatectomy. Many authors investigated relationship between urinary continence and preservation of bladder neck. Licht et al. reported in their study which includes 206 patients that preservation of bladder neck didn't have positive affect on urinary continence, but it was related to less bladder neck stenosis (20). Another study indicated that 24 patient who had bladder preserving radical prostatectomy had early recovery of urinary incontinence (21). When we look at these studies, the continence effect of preservation of internal sphincter is to passively keep urine above in the storage phase. In our study we didn't perform bladder neck preserving RRP and widely resected bladder neck with preservation of ureteral orifices. Preservation of puboprostatic ligaments is another topic which can be related to urinary incontinence. Some authors indicated positive effects of preservation of puboprostatic ligaments on post prostatectomy incontinence. Poore et al. preserved puboprostatic ligaments in 18 patients and compared them to control group which includes 25 patients (22). As a conclusion he founded early continence recovery in patients who underwent puboprostatic ligament preservation. In another study patients were divided into three groups. In first group bladder neck preserving surgery was done in second group puboprostatic ligament preserving surgery was done and to last group both bladder neck and puboprostatic preserving surgery was performed (23). There was no significant difference between three groups in long term urinary incontinence. But bladder neck preserving group had earlier urinary continence than ligament preserving group. In another study sling technique was performed by suturing bladder neck to pubic bone and significant earlier continence rates were indicated according to control group (24). In our study we didn't perform puboprostatic ligament preserving surgery. But we think that wide suturing in anterior urethra provides normal anatomic position of urethra and by this way it can help external sphincter functions by preventing urethral hypermobility. Lack of control group, randomization and the small number of patients included in this preliminary analysis could be considered as the main limitations of the present study.

\section{Conclusions}

Urinary incontinence after radical prostatectomy affects patients' quality of life and need to be treated early. Recovery of urinary continence depends on patient selection, surgical techniques and definition of conti- nence. We think that by modifications in our vesicourethral anastomosis technique; urethra is placed more anatomically on pelvic floor; caudal retraction of urethra is prevented and by this way functional urethral length stays long and especially wide suturing on anterior urethra sling the urethra anteriorly. According to our experience placing vesicourethral anastomotic suture along with rhabdospinchter is easy to perform. We also think that It is safe and shortens operation time and on the same time offers promising functional results. Multicentered, randomized controlled wide series is needed for these topics.

\section{REFERENCES}

1. Jemal A, Tiwari RC, Murray T, et al. Canser statistics. Canser J Clin. 2004; 54:8-29.

2. Siegel R, Naishadham D, Jemal A. Cancer statistics, 2013. CA Cancer J Clin. 2013; 63:11-30.

3. Walsh PC, Lepor H, Eggleston JC. Radical prostatectomy with preservation of sexual function: anatomical and pathological considerations. Prostate. 1983; 4:473-85.

4. Gandaglia G, Sammon JD, Chang SL, et al. Comparative effectiveness of robot-assisted and open radical prostatectomy in the postdissemination era. J Clin Oncol. 2014; 32:1419-26.

5. Kundu SD, Roehl KA, Eggener SE, et al. Potency, continence and complications in 3,477 consecutive radical retropubic prostatectomies. J Urol. 2004; 172:2227-31.

6. Walsh PC, Marschke P, Ricker D, Burnett AL. Patient-reported urinary continence and sexual function after anatomic radical prostatectomy. Urology. 2000; 55:58-61.

7. Arslan M, Tuncel A, Aslan Y, Kozacioglu Z, et al. Comparison of the urethrovesical anastomoses with polyglecaprone (Monocryl ${ }^{\circledR}$ ) and bidirectional barbed (V-Loc $180^{\circledR}$ ) running sutures in laparoscopic radical prostatectomy. Arch Ital Urol Androl. 2014; 86:90-4.

8. Kylmala T, Kaipia A, Matikainen M. Management of prolonged urinary leakage at the urethra-vesical anastomosis. Urol Int. 2005; 74:298-300

9. Castle EP, Andrews PE, Itano N, et al. Male sling postprostatectomy incontinence: Mean follow up 18 months. J Urology. 2005; 173:1657-60.

10. Sacco E, Prayer-Galetti T, Pinto F, et al. Urinary incontinence after radical prostatectomy: incidence by definition, risk factors and temporal trend in a large series with a long-term follow-up. BJU Int. 2006; 97:1234-41.

11. Lee SE, Byun SS, Lee HJ, et al. Impact of variations in prostatic apex shape on early recovery of urinary continence after radical retropubic prostatectomy. Urology. 2006; 68:137-41.

12. Burkhard FC, Kessler TM, Fleischmann A, et al. Nerve sparing open radical retropubic prostatectomy--does it have an impact on urinary continence? J Urol. 2006; 176:189-95.

13. Lepor $H$, Kaci $L$. The impact of open radical prostatectomy on continence and lower urinary tract symptoms: a prospective assessment using validated self-administered outcome instruments. J Urol. 2004; 171:1216-9.

14. Montorsi F, Salonia A, Suardi N, et al. Improving the preservation of the urethral sphincter and neurovascular bundles during open radical retropubic prostatectomy. Eur Urol. 2005; 48:938-45. 
15. Kim A, Kim M, Jeong SU, et al. Level of invasion into fibromuscular band is an independent factor for positive surgical marginand biochemical recurrence in men with organ confined prostate cancer. BMC Urol. 2018; 18:7.

16. Rocco F, Carmignani L, Acquati P, et al. Early continence recovery after open radical prostatectomy with restoration of the posterior aspect of the rhabdosphincter. Eur Urol. 2007; 52:376-83.

17. Eastham JA, Kattan MW, Rogers E, et al. Risk factors for urinary incontinence after radical prostatectomy. J Urol. 1996; 156:1707-13.

18. Myers RP. Male urethral sphincteric anatomy and radical prostatectomy. Urol Clin North Am. 1991; 18:211-27.

19. Mazaris EM, Chatzidarellis E, Varkarakis IM, et al. Reducing the number of sutures for vesicourethral anastomosis in radical retropubic prostatectomy. Int Braz J Urol. 2009; 35:158-63.
20. Licht MR, Klein EA, Tuason L, Levin H. Impact of bladder neck preservation during radical prostatectomy on continence and cancer control. Urology. 1994; 44:883-7.

21. Gaker DL, Gaker LB, Stewart JF, Gillenwater JY. Radical prostatectomy with preservation of urinary continence. J Urol. 1996; 156:445-9.

22. Poore RE, McCullough DL, Jarow JP. Puboprostatic ligament sparing improves urinary continence after radical retropubic prostatectomy. Urology. 1998; 51:67-72.

23. Deliveliotis C, Protogerou V, Alargof E, Varkarakis J. Radical prostatectomy: bladder neck preservation and puboprostatic ligament sparing-effects on continence and positive margins. Urology. 2002; 60:855-8.

24. Kojima Y, Hamakawa T, Kubota Y, et al. Bladder neck sling suspension during robot-assisted radical prostatectomy to improve early return of urinary continence: a comparative analysis. Urology. 2014; 83:632-9.

\section{Correspondence}

Ramazan Topaktaş, MD (Corresponding Author)

ramazantopaktas@yahoo.com

Ahmet Urkmez, MD

ahmeturkmez@hotmail.com

Musab Ali Kutluhan, MD

dr.musab151@hotmail.com

Haydarpaşa Numune Training and Research Hospital, Clinic of Urology,

İstanbul, Turkey

Tibbiye street No:23 - 34668 Uskudar/Istanbul

İsmail Başıbüyük, MD

dr.ismailbb@gmail.com

Beylikdüzü Kolan Hospital, Department of Urology, Istanbul, Turkey

Şinasi Yavuz Önol, MD

onolurology@yahoo.com

Onol Urology Center, Department of Urology, Istanbul, Turkey 